\title{
Entanglement in A Two-Identical-Particle System
}

\author{
Y.S. Li ${ }^{1,2}$, B. Zeng ${ }^{1,2}$, X.S. Liu $^{3}$, G.L. Long ${ }^{1,2}$ \\ 1. Department of Physics, Tsinghua University, Beijing, 100084, China \\ 2. Key Laboratory For Quantum Information and Measurements, Beijing, 100084, China \\ 3. Department of Physics, Shandong Normal University, Jinan, 250014, China
}

\begin{abstract}
The definition of entanglement in identical-particle system is introduced. The separability criterion in two-identical particle system is given. The physical meaning of the definition is analysed. Applications to two-boson and two-fermion systems are made. It is found new entanglement and correlation phenomena in identical-boson systems exist, and they may have applications in the field of quantum information.
\end{abstract}

It is no doubt that the phenomenon of quantum entanglement lies at the heart of the foundation of quantum mechanics. The original investigation on entanglement began from the famous EPR Gadanken experiment. Entanglement has been widely applied in many aspects of quantum information such as quantum teleportation, quantum cryptography and quantum computation. However, although it is well studied in distinguishable-particle systems, entanglement in identical-particle system has hardly been investigated, and even a proper definition is not given yet. It is noted that entanglement in certain system such as quantum dots [1], Bose-Einstein condensates [2] and parametric down conversion [3] must be dealt with in an identical particle manner. Recently this problem is noted by Schliemann et al [1, [] and they discussed the entanglement in two-fermion system. They have found that entanglement in two-fermion system is analogous to that in a two-distinguishable particle system, and the results obtained for two distinguishable-particle system can be translated into the two-fermion system. However due to the fundamental difference between bosons and fermions, the concept in two-boson system is quite different. In this paper, we explore the definition of entanglement in indistinguishable-particle systems. We first show that factorization alone is not able to define entanglement in a two-boson system. Then we gave a general definition for entanglement in identical particle systems. We show that this definition works well for two-boson system as well as for two-fermion system. Furthermore, the definition can be generalized into systems with more than two identical particles. We also address the concept of relative correlation.

Entanglement in distinguishable-particle system has been well studied. For a system of two distinguishable particles possessing single particle Hilbert space labeled by $\mathcal{H}_{1}$ and $\mathcal{H}_{2}$, the states can be described as vectors in the direct product space $\mathcal{H}_{1} \otimes \mathcal{H}_{2}$.

$$
|\Psi\rangle_{12}=\sum_{i, j} c_{i j}\left|\phi_{i}\right\rangle_{1} \otimes\left|\varphi_{j}\right\rangle_{2}
$$

where $\left\{\left|\phi_{i}\right\rangle\right\}$ is basis for $\mathcal{H}_{1}$ and $\left\{\left|\varphi_{j}\right\rangle\right\}$ is basis for $\mathcal{H}_{2}$ respectively. The state $|\Psi\rangle$ is called separable if and only if it can be written as $|\Psi\rangle_{12}=|\psi\rangle_{1} \otimes\left|\psi^{\prime}\right\rangle_{2}$, where $|\psi\rangle_{1} \in \mathcal{H}_{1}$ and $\left|\psi^{\prime}\right\rangle_{2} \in \mathcal{H}_{2}$, otherwise it is entangled. We can define local operations as those operations acting on $\mathcal{H}_{1}$ and $\mathcal{H}_{2}$ respectively. A separable state can't be transformed into an entangled state by any local operation and classical communication.

What will happen in indistinguishable-particle systems? Can the same definition be used? Firstly, Let's see the difference between distinguishable particle systems and identical systems. Suppose we have a two photon Bell state,

$$
|\leftrightarrow\rangle_{1}|\uparrow\rangle_{2}+|\uparrow\rangle_{1}|\leftrightarrow\rangle_{2}
$$

where $|\leftrightarrow\rangle$ and $|\uparrow\rangle$ stand for states with horizontal and vertical polarization respectively. If the two photons are separable, say the two photons have different momentum though their frequencies are the same. Then we can write state (2) in second quantization formalism as $\left(a_{1}^{\dagger} a_{3}^{\dagger}+a_{2}^{\dagger} a_{4}^{\dagger}\right)|0\rangle$, where $a_{1}^{\dagger}|0\rangle, a_{2}^{\dagger}|0\rangle, a_{3}^{\dagger}|0\rangle$ and $a_{4}^{\dagger}|0\rangle$ stands for single photon states $|\leftrightarrow\rangle_{1},|\uparrow\rangle_{1},|\leftrightarrow\rangle_{2}$ and $|\uparrow\rangle_{2}$ respectively. $|0\rangle$ is the vacuum state. Each photon in the system can be in one of the four modes $\left\{a_{i}^{\dagger}|0\rangle, i=1,2,3,4\right\}$, which span a 4-dimensional Hilbert space $\mathcal{H}=\mathcal{H}_{1} \oplus \mathcal{H}_{2} .\left(a_{1}^{\dagger} a_{3}^{\dagger}+a_{2}^{\dagger} a_{4}^{\dagger}\right)|0\rangle$ is not separable and thus it is entangled. However if the two photons are indistinguishable, then state (2) will be represented by $a_{\leftrightarrow}^{\dagger} a_{\uparrow}^{\dagger}|0\rangle$ which is separable, and hence not entangled.

In identical particle system, it is impossible to distinguish the two particles. A direct sum resolution of the single particle state into two constituent particle state is not possible. We can only say there is one particle in a given state, but we can not tell which of the two particles is in that state. Because of this, separable state in identical particle system may be defined, analogous to the case of distinguishable particles:

In identical two-particle system whose single particle Hilbert space $\mathcal{H}$ is span by $\alpha_{i}^{\dagger}|0\rangle i=1,2, \cdots, N$, a state is separable if it can be written as $c^{\dagger} d^{\dagger}|0\rangle$, where $c^{\dagger}|0\rangle, d^{\dagger}|0\rangle \in \mathcal{H}$. Otherwise it is entangled. 
It will be shown next that this definition does not cover all the entangled state in two-boson systems. The state $|\Psi\rangle$ of two identical bosons with single particle Hilbert space $\mathcal{H}=\mathcal{C}^{N}$ can be described as follow

$$
|\Psi\rangle=\sum_{i, j=1}^{N} \omega_{i j} a_{i}^{\dagger} a_{j}^{\dagger}|0\rangle
$$

where $\omega_{i j}=\omega_{j i}$ is an $N \times N$ complex symmetric matrix $\Omega$, and it can be decomposed to a diagonal matrix by the lemma below.

Lemma: For any symmetric $N \times N$ matrix $S$ there exists an unitary transformation $U$, such that $S=U D_{M} U^{T}$, where the matrix $D_{M}$ is diagonal,

$$
D_{M}=\operatorname{diag}\left[\mathrm{d}_{1}, \mathrm{~d}_{2}, \cdots, \mathrm{d}_{\mathrm{M}}, \mathrm{Z}\right]
$$

and $Z$ is a $(N-M) \times(N-M)$ null matrix. The proof of this lemma will be given in the appendix.

Now, we can diagonalize the state (3) by a unitary transformation $U$

$$
|\Psi\rangle=\sum_{j=1}^{M} \lambda_{j} c_{j}^{\dagger} c_{j}^{\dagger}|0\rangle, \quad c_{i}^{\dagger}=\sum_{j=1}^{N} u_{j i} a_{j}^{\dagger},
$$

where $U$ is a representation transformation and we arrange the eigenvalues in absolute value descending order $\left|\lambda_{1}\right| \geq$ $\left|\lambda_{2}\right| \geq \cdots \geq\left|\lambda_{M}\right|$. The above diagonal form can be regarded as a standard form because it is unique except for global phases in the definite of two-boson basis states. Since the rank of the matrix $\Omega, M$, does not change under unitary transformation, and it can be used as the criterion of entanglement for identical two-boson system.

If $N=2$, the standard form of (5) can be written as $\left(r_{1} e^{i \varphi} c_{1}^{\dagger} c_{1}^{\dagger}+r_{2} e^{-i \varphi} c_{2}^{\dagger} c_{2}^{\dagger}\right)|0\rangle$ after neglecting an overall phase factor. And $r_{1}$ and $r_{2}$ are nonnegtive. The state can be written as $\left[\left(r_{1}-r_{2}\right) f_{1}^{\dagger} f_{1}^{\dagger}+2 \sqrt{r_{1} r_{2}} f_{1}^{\dagger} f_{2}^{\dagger}\right]|0\rangle$ by a representation transformation,

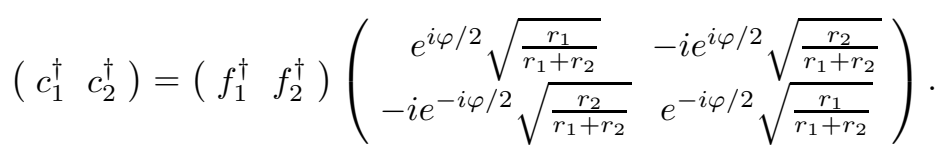

If $r_{1}=r_{2}$, the state will be $f_{1}^{\dagger} f_{2}^{\dagger}|0\rangle$ whose rank of coefficient matrix is 2 河. It would be a separable state if definition 1 is used.

It is easy to check that if (3) has the standard form as follows

$$
|\Psi\rangle=\sum_{i=1}^{L} z_{i}\left(e^{i \varphi_{i}} f_{1 i}^{\dagger} f_{1 i}^{\dagger}+e^{-i \varphi_{i}} f_{2 i}^{\dagger} f_{2 i}^{\dagger}\right)|0\rangle
$$

it can be transformed into $\sum_{i=1}^{L} 2 z_{i} c_{1 i}^{\dagger} c_{2 i}^{\dagger}|0\rangle$, which can be discussed as a system with distinguishable particles. If it has at least two nonzero $z_{i}$, it can be defined as distinguishable entangled state because it is identical to entangled states in distinguishable-particle systems. In general, a separable state according to definition 1 can be written as $c^{\dagger}\left(\alpha c^{\dagger}+\beta b \dagger\right)|0\rangle$ with the rank being either 1 or 2 , where $c^{\dagger}$ and $b^{\dagger}$ are orthogonal. States such as $c^{\dagger}\left(c^{\dagger}+b^{\dagger}\right)|0\rangle$ needs special attention. It has no invariant particle number in mode $c^{\dagger}, b^{\dagger}$ or $c^{\dagger}+b^{\dagger}$ and seems to have some persistent correlation. The density matrix of this state is not separable and it is an inseparable state. So the definition of entanglement has to be changed to let $c^{\dagger}\left(c^{\dagger}+b^{\dagger}\right)|0\rangle$ be entangled and hold states such as $c^{\dagger} d^{\dagger}|0\rangle$ and $c^{\dagger} c^{\dagger}|0\rangle$ as separable. It is worth pointing here $c^{\dagger} c^{\dagger}|0\rangle$ can be regarded as if it were a single particle, and we treat them as separable. Of course, the definition should be generalizable to identical multi-particle systems.

Now we can give the following definition of separability and entanglement in identical-particle systems:

Definition 2 A state with identical k-particle is separable if it can be written as $c_{1}^{\dagger} c_{2}^{\dagger} \cdots c_{k}^{\dagger}|0\rangle$, where $c_{i}^{\dagger}$ and $c_{j}^{\dagger}$ are either equal or orthogonal. Otherwise it is entangled.

This definition works for both identical boson system and identical fermion system, because in the fermion system, Pauli principle prohibit two particles to occupy the same state, states with $c^{\dagger}\left(c^{\dagger}+b^{\dagger}\right)|0\rangle=c^{\dagger} b^{\dagger}|0\rangle$ which becomes the product of two orthogonal states automatically. It is interesting to point out that an equivalent definition can be formulated in the following: a state with identical $k$-particle is separable if it is an eigenvector of a complete set of one-body hermitian operators, otherwise it is entangled. Complete operator sets can be generated by the operators $\left\{\left(a_{i}^{\dagger} a_{j}+a_{j}^{\dagger} a_{i}\right) / 2, i, j=1,2, \cdots, n\right\}$. This alternative definition is consistent with the statement in [6, 7 , which says 
that any basis that is eigenvectors of complete set consists of one-body mechanical quantities must be separable in distinguishable-particle case.

For systems with 2 bosons, we have standard form (2) to tell whether a state is entangled or not. And a state must be entangled if the rank of its coefficient matrix $\Omega$ is greater than 2 . If $\operatorname{rank}(\Omega)$ is 2 , it is also easy to judge whether it is entangled or not from its standard form according to definition 2. From a normalized standard form like (5), we can define the entanglement measure as

$$
-4 \sum_{i=1}^{[M / 2]}\left(\left|\lambda_{2 i-1} \lambda_{2 i}\right|\right) \ln \left(\left|4 \lambda_{2 i-1} \lambda_{2 i}\right|\right)
$$

for a two-boson systems. It is similar to that in distinguishable-particle systems and if the standard form can be written as (7) which has counterpart in distinguishable system, the entanglement measure thus defined will be just the partial entropy in distinguishable-particle systems [11]. But for systems with $k \mathrm{k}(k>2)$ bosons, it is more difficult to give the standard forms to tell whether a state is separable. It is also noted that the quantitative description of entanglement in multi-particle systems is a very hard mathematical problem, and it is still not solved in distinguishable-particle systems. The problem to quantify entanglement in identical-boson systems is even more difficult and remains an open challenge.

New entanglement has been found in identical boson systems. They may have important applications different from those in distinguishable-particle systems. For example $\left(a^{\dagger} a^{\dagger}+b^{\dagger} c^{\dagger}\right)|0\rangle$ is an entangled state in an identical boson system with $N=3$ single particle space. It can denote a superposed state of two photons states, one with two photons sent to Alice, and one with one photon sent to Bob and the other sent to Clare. If Alice, Bob and Clare measure photon number respectively. If Alice gets two photons, none photon will reach Bob and Clare, and if no photon reach Alice, either of Bob and Clare will have one photon. If Bob get one photon, the other photon will reach Clare. Situation of Clare is similar to Bob. So if one person of the three get a result, the results of the other two are decided, which means that some kind of communication can be built with only two photons.

The above definition of entanglement also applies to many identical fermion system. Schliemann et al have discussed entanglement in two-fermion systems [4]. For a two-fermion system having the single particle Hilbert space $\mathcal{C}^{2 K}$, an arbitrary state has the form $|\Psi\rangle=\sum_{i, j=1}^{2 K} \omega_{i j} a_{i}^{\dagger} a_{j}^{\dagger}|0\rangle$, where $\Omega=\left(\omega_{i j}\right)$ are antisymmetric. It can be decomposed into a standard form $\sum_{i}^{K} z_{i} f_{1 i}^{\dagger} f_{2 i}^{\dagger}|0\rangle$ by a representation transformation 清, which implies that entanglement and separability in two-fermion systems are equivalent with those in distinguishable-particle systems. However when generalizing this into many fermion system, this elegant property in two-fermion system disappear in many fermion system. This can be easily understood that the single particle Hilbert space $\mathcal{H}$ for a state in system with $k$ greater than 2 identical fermions can't be decomposed to a direct sum resolution of $\mathrm{k}$ subspaces. For example, composing a three-fermion state with single particle Hilbert space $\mathcal{C}^{3 N}$ to three orthogonal N-dimension subspaces requires $7 N^{3}-9 N^{2}+2 N$ real equations satisfied while the group $S U(3 N)$ can only provide $9 N^{2}-1$ real parameters. The equations have the required number of parameters only when $N=1$ or $N=2$. It is easy to check the definition 1 and definition 2 are equivalent for identical fermion system because arbitrary two fermions can't be in one state. Hence for identical two-fermion systems, the rank of $\Omega$ is the criterion to judge entanglement or separability: $\operatorname{rank}(\Omega)=2$ for separability and $\operatorname{rank}(\Omega)>2$ for entanglement. It must be noted that $\operatorname{rank}(\Omega) \neq 1$ for two-fermion systems.

Another important concept is quantum correlation. It is quite often used in literatures of physics. A recent development has made this concept an important one. It is well known that quantum teleportation can be implemented with Bell states which are distinguishably entangled. Lee et al gave an experimental scheme, in which a state superposed by one photon and vacuum can be teleported with a single photon state $\left(a^{\dagger}+b^{\dagger}\right)|0\rangle$ [ [ ] , where $a^{\dagger}$ and $b^{\dagger}$ are particle creation operators in path A and B. It is meaningless to discuss entanglement for a single particle, therefore there is not entanglement in this teleportation scheme. They suggests that entanglement may be not necessary for quantum teleportation, because it can even be implemented with separable states. To study phenomenon like this, it is useful to define relative correlation:

Definition 3 A state is said to have correlation relative to a quantum-mechanical quantity $F$, if and only if the state is not an eigenvector of $F$.

According to the definition above, the quantity $F$ is important for certain correlation. A correlation must be related to certain measurement corresponding to the mechanical quantity $F$. In fact, the so-called correlation is the correlation between eigenvectors with different eigenvalues of $F$. Operations in eigenvectors with the same eigenvalue may be called local operations. Operations in eigenvectors with different eigenvalues are nonlocal. It is obvious that local operations do not change eigenvectors' eigenvalues. There is correlation relative to particle number $a^{\dagger} a$ or $b^{\dagger} b$ in $\left(a^{\dagger}+b^{\dagger}\right)|0\rangle$ and no correlation relative to the two particle number operators in $a^{\dagger} b^{\dagger}|0\rangle$, where $a^{\dagger}$ and $b^{\dagger}$ are orthogonal. For the state $a^{\dagger} b^{\dagger}|0\rangle$, local operations relative to $a^{\dagger} a$ do not affect the particle at mode $b^{\dagger}$, and the similar occurs 
to $b^{\dagger} b$. Hence $a^{\dagger} b^{\dagger}|0\rangle$ is called a separated state in distinguishable-particle systems. Entanglement must have some correlation, but correlation can happen in both separable and entangled states.

To summarize, entanglement in identical particle system can be well distinghuished by definition 2 . This definition reduces to that in distinguishable-particle system if the particles are distinguishable. It is noted that the entanglement definition of both distinguishable-particle and identical-particle systems can be dealt with using the definition given in this paper. Using identical particle formalism to treat identical particle system is important, examples of such treatment can be found in Refs. [9 10 . There are also new phenomena in identical-particle systems, which may have future applications. Moreover, relative correlation is defined, and it may be physically more important than entanglement.

\section{PROOF OF LEMMA}

Proof: Let $S$ be a $N \times N$, complex, symmetric matrix, $S^{T}=S$. Hence $S S^{*}=S S^{\dagger}$ is hermitian which can be diagonalized by a unitary transformation $U^{\prime}: S S^{*}=U^{\prime} D U^{\prime \dagger}$, D - diagonal. Let us define $C:=U^{\prime \dagger} S U^{\prime *}$. It is easy check that $\mathrm{C}$ is symmetric and normal $C C^{\dagger}=C^{\dagger} C$. Then we'll decompose $C$ into its real and imaginary parts: $C=F+i G$. Since $\mathrm{C}$ is normal, $\mathrm{F}$ and $\mathrm{G}$ commute. Thus $F$ and $G$ are real, symmetric, commuting matrices. Hence they can be simultaneously diagonalized by a real orthogonal transformation $O, F=O D^{1} O^{T}$ and $G=O D^{2} O^{T}$. Thus $C=O D_{M} O^{T}\left(D_{M}=D^{1}+D^{2}\right)$ and finally

$$
S=U^{\prime} O D_{M} O^{T} U^{T}=U D_{M} U^{T}
$$

where $U=U^{\prime} O$.

\section{ACKNOWLEDGEMENT}

The authors would like to thank Prof. J.Y. Zeng, Prof. C.P. Sun, Prof. S.Y. Pei, Mr. P. Zhang and Mr. H. Zhai for useful discussions. This work is supported by the China National Natural Science Foundation Grant No. 60073009, the Fok Ying Tung Education Foundation, and the Excellent Young University Teachers' Fund of Education Ministry of China.

[1] J. Schliemann, D. Loss and A.H. MacDonald, Phys. Rev. B63(2001)085311

[2] A. S $\phi$ rensen, L.M. Duan, J.I. Cirac and P. Zoller, Natrue 409(2001)63.

[3] Y.H. Kim, M.V. Chekhova, S.D. Knlik, M.H. Rubin and Y. Shih, to appear in Phys. Rev. A. Also available in quant$\mathrm{ph} / 0103168$.

[4] J. Schliemann, J.. Cirac, M. Kuús, M. Lewenstein and D. Loss, quant-ph/0012094.

[5] $f_{1}^{\dagger} f_{2}^{\dagger}|0\rangle=\sum_{i, j} \omega_{i, j} f_{i}^{\dagger} f_{j}^{\dagger}=\left(f_{1}^{\dagger} f_{2}^{\dagger}|0\rangle+f_{2}^{\dagger} f_{1}^{\dagger}|0\rangle\right) / 2$

[6] H.B. Zhu and J.Y. Zeng, to be published in Science in China.

[7] H.B. Zhu and J.Y. Zeng, to be published in Chin. Phys. Lett.

[8] H.W. Lee and J. Kim, Phys. Rev. A.63(2000)012305

[9] J. Calsamiglia and N. Lütkenhaus, Appl. Phys. B 72(2001) 67. Also in quant-ph/0007058.

[10] N. Lütkenhaus, J. Calsamiglia and K.A. Suominen, Phys. Rev. A59(1999)3295.

[11] C.H. Bennett, D.P. Divincenzo, J.A. Smolin and W.K. Wootters, Phys. Rev. A54(1996)3824, quant-ph/9604024 